\title{
Reducción del contenido de alcohol y pH de vinos tintos Pinot noir y Tannat empleando uvas con diferentes niveles de maduración
}

\section{Reduction of the alcohol content and pH of Pinot noir and Tannat red wines using grapes with different maturation level}

\author{
D. Piccardo ${ }^{1, a}$, G. Favre ${ }^{1}$, O. Pascual ${ }^{2}$, J.M. Canals ${ }^{2}$, F. Zamora² ${ }^{2}$ y G. González-Neves ${ }^{1}$ \\ ${ }^{1}$ Unidad de Tecnología de los Alimentos, Facultad de Agronomía. UDELAR. Garzón 780, Montevideo, Uruguay \\ 2 Departament de Bioquímica i Biotecnologia, Facultat d'Enologia de Tarragona, Grup de Recerca en Tecnologia Enològica \\ (TECNENOL), Universitat Rovira i Virgili, Campus de Sescelades, C/Marcel.li Domingo s/n, 43007 Tarragona, Spain
}

\begin{abstract}
Resumen. El objetivo de esta investigación fue determinar la efectividad de la mezcla de mostos de uvas con diferente grado de maduración para la obtención de vinos Pinot noir y Tannat con menor contenido de alcohol y pH. En envero, se extrajo mosto de uvas inmaduras que se conservó a $4{ }^{\circ} \mathrm{C}$ hasta su uso. En madurez tecnológica, las uvas cosechadas fueron despalilladas, estrujadas y distribuidas en seis recipientes. Tres fueron vinificados de forma tradicional, mientras que en los otros tres se realizó una sustitución de 3 L del mosto de uva madura por $3 \mathrm{~L}$ del mosto de uvas inmaduras. Ambos tratamientos realizaron una maceración fermentativa de 7 días. Se determinó la composición básica del vino, color, concentración de fenoles totales, antocianos, catequinas y proantocianidinas a los dos meses del descube. La sustitución de mosto permitió elaborar vinos Pinot noir y Tannat con menor contenido de alcohol y pH, sin reducir las concentraciones de polifenoles totales, catequinas o antocianos. El efecto sobre la concentración de proantocianidinas fue variable de acuerdo con el cultivar y año de vendimia. Los vinos Tannat reducidos en alcohol presentaron mayor intensidad de color, menor tono e igual cromaticidad que los vinos testigos.
\end{abstract}

\begin{abstract}
The aim of this research was to determine the effectiveness of the mixture of the must of grapes with different degree of maturation to obtain Pinot Noir and Tannat wines with lower alcohol content and $\mathrm{pH}$. In veraison, immature grape must was extracted and kept at $4{ }^{\circ} \mathrm{C}$ until its use. In technological maturity, the grapes harvested were destemmed, crushed and distributed in six containers. Three of them were vinified in a traditional way, while in the other three, $3 \mathrm{~L}$ of the mature grape must was substituted for $3 \mathrm{~L}$ of the immature grape must. Both treatments performed a fermentative maceration of 7 days. The basic wine composition, color, concentration of total phenols, anthocyanins, catechins and proanthocyanidins were determined two months after devatting. The substitution of must allowed the production of Pino noir and Tannat wines with lower alcohol content and $\mathrm{pH}$ without reducing the concentrations of total polyphenols, catechins or anthocyanins. The effect on the concentration of proanthocyanidins was variable according to the cultivar and year of harvest. The Tannat wines reduced in alcohol presented greater intensity of color, lower hue and similar chromaticity than the control wines.
\end{abstract}

\section{Introducción}

Los vinos tintos con mucho cuerpo y de color intenso son muy apreciados por el mercado. Su producción requiere una intensa extracción de compuestos fenólicos durante la vinificación, proceso afectado por la madurez de la uva $[1,2]$ y razón por la que se busca cosecharla lo más madura posible. Las uvas con madurez muy avanzada presentan altas concentraciones de azúcares y baja acidez $[3,4]$. Frente a estas circunstancias se pueden tomar dos decisiones diferentes de cosecha. Por un lado, cosechar uvas con un contenido de azúcares y $\mathrm{pH}$ adecuados, pero con una maduración inadecuada de hollejos y semillas que

a e-mail: dpiccardo@fagro.edu.uy probablemente originan vinos mal coloreados, amargos, astringentes y herbáceos. Por otro lado, se puede esperar la madurez fenólica completa y suponer que sus vinos tendrán el inconveniente de un $\mathrm{pH}$ y un contenido de alcohol elevados [5]. Adicionalmente, el calentamiento global está acentuando esta tendencia en los últimos años $[6,7]$.

Las recientes políticas sociales llevadas a cabo por muchos países han puesto de manifiesto la necesidad de regular el consumo de bebidas alcohólicas a corto plazo. Como resultado de la aplicación de estas medidas sumadas a las preocupaciones relacionadas con la salud y los comportamientos sociales de la población, hay una disminución en el consumo de bebidas con altos niveles de alcohol. El vino es una de las bebidas 
alcohólicas más consumidas. Varias investigaciones han informado que el consumo moderado de vino tinto puede tener efectos beneficiosos para la salud debido a los contenidos significativos de compuestos bioactivos [8]. Los compuestos bioactivos del vino son los estilbenos, antocianos, taninos y otros polifenoles cuya relevancia está determinada por sus características tecnológicas, sensoriales y nutricionales [3,9]. Estos compuestos tienen capacidad antioxidante y participan en numerosos procesos bioquímicos potencialmente positivos para el organismo. Reducir el contenido de alcohol sin modificar el resto de los componentes del vino es uno de los objetivos actuales perseguidos por la industria [10].

Varias técnicas de vinificación han sido propuestas para elaborar vinos con menor contenido de alcohol, sin alterar su composición fenólica. Entre ellas destacan: la cosecha de uvas en una etapa temprana de maduración [11], el agregado de agua y ácidos minerales al mosto antes del inicio de la fermentación [12], la introducción de nuevos cultivares y la modificación de las técnicas de manejo del viñedo [13], el uso de glucosa oxidasa (EC 1.1.3.4) [14], el uso de levaduras con un menor rendimiento en producción de etanol [15] y la aplicación de técnicas físicas para reducir parcialmente la concentración de azúcares en el mosto o el alcohol en el vino [16-18]. En ese sentido, la sustitución de un parte del mosto de uvas muy maduras por mosto de uvas verdes previamente fermentado [19] o por mosto previamente tratado por osmosis inversa [20] han sido procedimientos propuestos reducir simultáneamente el contenido de etanol y $\mathrm{pH}$ de vino.

El objetivo de esta investigación fue determinar la efectividad de la mezcla de mostos de uvas con diferente grado de maduración para obtener vinos tintos Pinot noir y Tannat con menor contenido de alcohol y pH, sin modificar su color y composición fenólica.

\section{Materiales y métodos}

\subsection{Cosecha y vinificaciones}

Los vinos se elaboraron durante la vendimia 2016, 2017 y 2018 con uvas del cultivar Pinot noir (Vitis vinifera L., Vitis International Variety Cataloque number: VIVC 9279) [21] y Tannat (Vitis vinifera L., Vitis International Variety Catalogue number: VIVC 12257) [21] cosechadas de viñedos comerciales ubicados en Canelón Chico, Canelones, Uruguay.

Al comienzo del envero, se cosecharon $100 \mathrm{~kg}$ de uva de cada cultivar para obtener un mosto con baja concentración de azúcares y alta acidez. Las uvas se despalillaron, estrujaron (Alfa 60 R, Italcom, Piazzola Sul Brenta, Italia) y se prensaron ligeramente en una prensa manual hasta obtener $50 \mathrm{~L}$ de mosto inmaduro. Este mosto se sulfitó con $100 \mathrm{mg} / \mathrm{L}$ de $\mathrm{K}_{2} \mathrm{~S}_{2} \mathrm{O}_{2}$, se sedimentó durante la noche, se envasó en un recipiente de $50 \mathrm{~L}$ de capacidad y se conservó a $4{ }^{\circ} \mathrm{C}$ hasta su uso. Cuando las uvas alcanzan la madurez tecnológica, se cosecharon $120 \mathrm{~kg}$ de uvas de ambos cultivares y se distribuyeron aleatoriamente en seis lotes de $10 \mathrm{~kg}$ cada uno por cultivar. Las uvas fueron despalilladas y estrujadas (Alfa 60 R, Italcom, Piazzola Sul Brenta, Italia) y el mosto se sulfitó con $100 \mathrm{mg} / \mathrm{L}$ de $\mathrm{K}_{2} \mathrm{~S}_{2} \mathrm{O}_{2}$. Los contenedores de mostos se dividieron aleatoriamente en dos grupos de tres contenedores cada uno. Tres contenedores se consideraron como testigos (mosto testigo - MT) mientras que en los otros tres contenedores se sustituyeron 31 de mosto original por 31 del mosto inmaduro con baja concentración de azúcares y $\mathrm{pH}$ (mosto reducidos en azúcares - MRA).

Los mostos de ambos tratamientos fueron sometidos a una maceración fermentativa de 7 días. Todos los tanques se inocularon con $200 \mathrm{mg} / \mathrm{L}$ de levadura seca activa (Saccharomyces cerevisiae ex bayanus Natuferm 804; Oenobiotech, Paris, Francia). Durante la maceración, se realizó un remontaje diario a fin de favorecer la extracción de polifenoles. La temperatura de fermentación estuvo comprendida entre 25 y $29^{\circ} \mathrm{C}$. Después de 7 días de maceración, el vino se extrajo por gravedad y los orujos se prensaron ligeramente en una prensa manual. El vino se mantuvo en recipientes de $5 \mathrm{~L}$ de capacidad a temperatura ambiente. Una vez finalizada la fermentación maloláctica espontánea, todos los vinos se estabilizaron con $100 \mathrm{mg} / \mathrm{l}$ de $\mathrm{K}_{2} \mathrm{~S}_{2} \mathrm{O}_{2}$ y $300 \mathrm{mg} / \mathrm{l}$ de lisozima (DelvoßZyme, Delft, Países Bajos). Finalmente, los vinos fueron embotellados y almacenados hasta su análisis. Los análisis se realizaron a los 2 meses del descube.

\subsection{Análisis de la composición general de las uvas y los vinos elaborados}

Los métodos analíticos recomendados por la Organización Internacional de la Viña y el Vino [22] se utilizaron para determinar la concentración de azúcares, el pH y la acidez titulable de los mostos. El contenido total de antocianinas de las uvas, su extractibilidad y riqueza fenólica se determinó de acuerdo con el método de Glories y Augustien [23] modificado por González- Neves et al. [24].

El contenido de etanol, la acidez titulable y el $\mathrm{pH}$ de los vinos se determinaron a los dos meses del descube utilizando el analizador de infrarrojos Winescan TM Autosampler 79000 (Foss, EE. UU.) y el software Foss Integrator versión 154 (Foss, Dinamarca).

\subsection{Determinación de los parámetros cromáticos}

Los parámetros de color se determinaron directamente en las muestras de vino colocadas en una cubeta de $1 \mathrm{~mm}$ de recorrido óptico. La intensidad colorante (IC) se estimó utilizando el método descrito por Glories [25]. Las coordenadas CIELAB, luminosidad $\left(\mathrm{L}^{*}\right)$, cromaticidad $\left(\mathrm{C}^{*}\right)$ y tono $\left(\mathrm{H}^{*}\right)$, se determinaron de acuerdo con el método de Ayala et al. [26] y el procesamiento de datos se realizó utilizando el software MSCV [27].

\subsection{Análisis espectrofotométricos de la composición fenólica de los vinos y parámetros relacionados}

Los análisis espectrofotométricos de los vinos se realizaron a los dos meses del descube.

La composición polifenólica se evaluó utilizando índices clásicos espectrofotométricos. Los polifenoles totales se determinaron usando el Folin-Ciocalteu, de acuerdo con Singleton y Rossi [28], y sus contenidos en los vinos se expresan en mg de ácido gálico por litro. La 
Tabla 1. Composición básica de las uvas según cultivar y momento de cosecha.

\begin{tabular}{|c|c|c|c|c|c|c|c|c|}
\hline Cultivar & $\begin{array}{c}\text { Año de } \\
\text { vendimia }\end{array}$ & $\begin{array}{l}\text { Momento de } \\
\text { cosecha }\end{array}$ & $\begin{array}{c}\text { Azúcares } \\
(\mathrm{g} / \mathrm{L})\end{array}$ & $\begin{array}{l}\text { Acidez titulable } \\
\left(\mathrm{gH}_{2} \mathrm{SO}_{4} / \mathrm{L}\right)\end{array}$ & pH & A280 & ApH1 & EA (\%) \\
\hline \multirow{6}{*}{ Pinot Noir } & \multirow[t]{2}{*}{2016} & 1 $^{\mathrm{er}}$ cosecha & $129 \pm 1 b$ & $12.25 \pm 0.09 \mathrm{a}$ & $3.10 \pm 0.05 b$ & - & - & - \\
\hline & & $2^{\text {da }}$ cosecha & $243 \pm 1 \mathrm{a}$ & $3.62 \pm 0.18 b$ & $3.52 \pm 0.10 \mathrm{a}$ & $33.5 \pm 3.7$ & $939 \pm 18$ & $30.8 \pm 1.8$ \\
\hline & \multirow[t]{2}{*}{2017} & $1^{\mathrm{er}}$ cosecha & $171 \pm 1 \mathrm{~b}$ & $5.11 \pm 0.04 \mathrm{a}$ & $3.29 \pm 0.01 \mathrm{~b}$ & - & - & . \\
\hline & & $2^{\mathrm{da}}$ cosecha & $224 \pm 21 \mathrm{a}$ & $4.86 \pm 0.03 \mathrm{~b}$ & $3.45 \pm 0.02 \mathrm{a}$ & $53.4 \pm 3.3$ & $212 \pm 2.3$ & $26.0 \pm 1.8$ \\
\hline & \multirow[t]{2}{*}{2018} & $1^{\mathrm{er}} \operatorname{cosech} \mathrm{a}$ & $175 \pm 2 b$ & $4.48 \pm 0.10 \mathrm{a}$ & $3.22 \pm 0.01 \mathrm{~b}$ & - & - & - \\
\hline & & $2^{\mathrm{da}}$ cosecha & $245 \pm 3 \mathrm{a}$ & $3.94 \pm 0.17 b$ & $3.59 \pm 0.04 \mathrm{a}$ & $58.9 \pm 3.8$ & $434.5 \pm 41.3$ & $25 \pm 1.2$ \\
\hline \multirow{6}{*}{ Tannat } & \multirow[t]{2}{*}{2016} & $1^{\mathrm{er}} \operatorname{cosecha}$ & $175 \pm 2 b$ & $8.43 \pm 0.12 b$ & $3.12 \pm 0.04 b$ & - & - & - \\
\hline & & $2^{\mathrm{da}} \operatorname{cosech} \mathrm{a}$ & $243 \pm 3 \mathrm{a}$ & $4.51 \pm 0.07 \mathrm{a}$ & $3.31 \pm 0.03 \mathrm{a}$ & $47.5 \pm 0.5$ & $2258 \pm 145$ & $51.6 \pm 2.6$ \\
\hline & \multirow[t]{2}{*}{2017} & $1^{\mathrm{er}}$ cosecha & $182 \pm 2 b$ & $5.98 \pm 0.05 \mathrm{a}$ & $3.10 \pm 0.02 b$ & - & - & - \\
\hline & & $2^{\mathrm{da}} \operatorname{cosech} \mathrm{a}$ & $193 \pm 1 \mathrm{a}$ & $5.48 \pm 0.07 \mathrm{~b}$ & $3.23 \pm 0.03 \mathrm{a}$ & $61.5 \pm 1.5$ & $949 \pm 3.0$ & $49.2 \pm 0.6$ \\
\hline & \multirow[t]{2}{*}{2018} & $1^{\mathrm{er}} \operatorname{cosecha}$ & $191 \pm 1 b$ & $3.63 \pm 0.05 b$ & $3.14 \pm 0.03 b$ & - & - & - \\
\hline & & $2^{\text {da }} \operatorname{cosecha}$ & $263 \pm 6 a$ & $4.19 \pm 0.27 \mathrm{a}$ & $3.27 \pm 0.01 \mathrm{a}$ & $81.6 \pm 3.4$ & $2334.5 \pm 19.3$ & $47.0 \pm 1.1$ \\
\hline
\end{tabular}

Medias con distinta letra indican diferencias significativas $(p<0.05)$. A280: riqueza fenólica de la uva, ApH1: potencial en antocianos totales, EA $(\%)$ : es índice de extractibilidad de los antocianos.

concentración de antocianos fue analizada por RibéreauGayon y Stonestreet [29] y se expresa en mg de malvidin3-glucósido equivalente (EMG) por litro. Las catequinas se cuantificaron usando el método de Swain y Hillis [30] y sus concentraciones se expresan en mg de D-catequina por litro. Las proantocianidinas se determinaron según Ribéreau-Gayon y Stonestreet [29] y sus contenidos se expresan en mg de cloruro de cianidina por litro de vino.

\subsection{Análisis estadistico}

Todos los datos se expresan como el promedio aritmético \pm desviación estándar de tres repeticiones. Las diferencias estadísticas entre los tratamientos fueron determinadas aplicando análisis de varianza multivariados. Los análisis estadísticos se realizaron utilizando el software Infostat versión 2015 [31].

\section{Resultados y discusión}

\subsection{Composición de los mostos}

La Tabla 1 muestra la concentración de azúcares, la acidez titulable y el pH de los mostos Pinot noir y Tannat cosechados a principios del envero $\left(1^{\mathrm{er}} \operatorname{cosecha}\right)$ y en madurez tecnológica ( $2^{\text {da }}$ cosecha) durante las vendimias 2016, 2017 y 2018. Como era de esperar, la concentración de azúcares y el pH aumentaron durante la maduración de las uvas, mientras que la acidez titulable disminuyó.

Los parámetros indicadores del potencial fenólico de la uva se determinaron en cada año, solamente en las uvas de la $2^{\text {da }}$ cosecha ya que solo los hollejos y semillas de las uvas cosechadas en madurez tecnológica aportaron compuestos fenólicos durante la maceración. Como se esperaba, las uvas Tannat presentaron un mayor potencial en compuestos fenólicos totales (A280) y antocianos totales (ApH1) que las uvas Pinot Noir. Sin embargo, el índice de extractabilidad de los antocianos (EA\%) fue mayor en las uvas Tannat que en las uvas Pinot Noir, lo que indica que la proporción de antocianos que se liberarán durante la maceración será menor en las uvas Tannat que en las uvas Pinot Noir. Estos resultados están de acuerdo con las características descritas para estas variedades en Uruguay [24].

Asimismo, el potencial enológico de las uvas fue diferente de acuerdo con las condiciones de maduración de cada año. Tanto las uvas Tannat como Pinot noir presentaron mayor concentración de azúcares, potencial en compuestos fenólicos totales y antocianos totales, y menor índice de extractibilidad de los antocianos en la vendimia 2018. En contrapartida, el menor potencial enológico de las uvas se registró en la vendimia 2017 para ambos cultivares. Investigaciones realizadas en nuestras condiciones climáticas por Ferrer et al. [32] demuestran que los compuestos relacionados con la calidad de la uva se ven favorecidos por la acumulación de temperaturas durante las primeras etapas del ciclo de cultivo, mientras que las altas temperatura y la disponibilidad hídrica durante la maduración los afectan negativamente. Estas condiciones son muy variables entre años, lo cual explicaría las diferencias registradas en el potencial enológico de las uvas entre vendimias.

\subsection{Composición básica de los vinos}

La Tabla 2 muestra el contenido de etanol, la acidez total y el $\mathrm{pH}$ de los vinos Pinot noir y Tannat elaborados durante las vendimias 2016, 2017 y 2018.

En ambos cultivares, el contenido de etanol y el $\mathrm{pH}$ de los vinos elaborados con mosto testigo (MT) fue significativamente superior al de los vinos elaborados a partir de mostos reducidos en azúcares (MRA) con la única excepción del Tannat 2017 donde no se observaron diferencias significativas en el $\mathrm{pH}$. La acidez total fue menor para los vinos testigo de ambos cultivares, si bien no se observaron diferencias significativas entre los tratamientos en todas las vinificaciones.

Estos resultados eran esperables ya que la sustitución de mosto de uva en madurez tecnológica por mosto de uvas inmaduras implica una disminución en el contenido de azúcares y un aumento de la acidez. Por ende, los vinos elaborados con esta técnica de vinificación presentan vinos con menor contenido de alcohol y $\mathrm{pH}$. Estos resultados están de acuerdo con los obtenidos por Kontoudakis et al. 
Tabla 2. Composición básica de los vinos según cultivar y año de vendimia.

\begin{tabular}{|c|c|c|c|c|c|}
\hline Cultivar & $\begin{array}{l}\text { Año de } \\
\text { vendimia }\end{array}$ & $\begin{array}{l}\text { Composición } \\
\text { del mosto }\end{array}$ & $\begin{array}{l}\text { Alcohol } \\
(\% \mathrm{v} / \mathrm{v})\end{array}$ & $\begin{array}{l}\text { Acidez Total } \\
\left(\mathrm{gH}_{2} \mathrm{SO}_{4} / \mathrm{L}\right)\end{array}$ & pH \\
\hline \multirow{6}{*}{ Pinot noir } & \multirow[t]{2}{*}{2016} & MT & $14.3 \pm 0.1 \mathrm{a}$ & $3.11 \pm 0.04 b$ & $3.77 \pm 0.02 \mathrm{a}$ \\
\hline & & MRA & $13.5 \pm 0.1 \mathrm{~b}$ & $5.42 \pm 0.06 \mathrm{a}$ & $3.38 \pm 0.01 b$ \\
\hline & \multirow[t]{2}{*}{2017} & MT & $13.2 \pm 0.1 \mathrm{a}$ & $2.60 \pm 0.04 \mathrm{a}$ & $3.91 \pm 0.02 \mathrm{a}$ \\
\hline & & MRA & $12.2 \pm 0.1 \mathrm{~b}$ & $2.65 \pm 0.05 \mathrm{a}$ & $3.84 \pm 0.03 b$ \\
\hline & \multirow[t]{2}{*}{2018} & MT & $15.7 \pm 0.2 \mathrm{a}$ & $3.09 \pm 0.18 b$ & $3.90 \pm 0.02 \mathrm{a}$ \\
\hline & & MRA & $13.7 \pm 0.1 \mathrm{~b}$ & $3.30 \pm 0.03 \mathrm{a}$ & $3.68 \pm 0.02 b$ \\
\hline \multirow{6}{*}{ Tannat } & \multirow[t]{2}{*}{2016} & MT & $14.6 \pm 0.2 \mathrm{a}$ & $3.88 \pm 0.21 b$ & $4.01 \pm 0.06 \mathrm{a}$ \\
\hline & & MRA & $13.1 \pm 0.1 \mathrm{~b}$ & $4.51 \pm 0.84 \mathrm{a}$ & $3.85 \pm 0.04 b$ \\
\hline & \multirow[t]{2}{*}{2017} & MT & $11.5 \pm 0.2 \mathrm{a}$ & $3.02 \pm 0.01 \mathrm{a}$ & $3.82 \pm 0.01 \mathrm{a}$ \\
\hline & & MRA & $10.9 \pm 0.2 b$ & $3.07 \pm 0.04 \mathrm{a}$ & $3.83 \pm 0.04 a$ \\
\hline & \multirow[t]{2}{*}{2018} & MT & $16.1 \pm 0.1 \mathrm{a}$ & $3.93 \pm 0.05 \mathrm{a}$ & $3.93 \pm 0.01 \mathrm{a}$ \\
\hline & & MRA & $13.8 \pm 0.1 \mathrm{~b}$ & $4.04 \pm 0.02 \mathrm{a}$ & $3.75 \pm 0.02 b$ \\
\hline
\end{tabular}

Medias con distinta letra indican diferencias significativas $(p<0.05)$. MT: vino elaborado con mosto testigo; MRA: vino elaborado con mosto reducido en azúcares.

[19] y Rolle et al. [20] en el que han aplicado una técnica de sustitución de mosto de uvas maduras.

\subsection{Composición fenólica de los vinos}

La concentración de fenoles totales, antocianos, catequinas y proantocianidinas para los vinos Pinot noir y Tannat elaborados durante la vendimia 2016, 2017 y 2018 se observa en la Tabla 3.

En general, la concentración de compuestos fenólicos totales de los vinos Tannat y Pinot noir no fue afectada por la composición inicial del mosto, con la única excepción del Tannat 2016 donde la diferencia entre los tratamientos fue de un $9.3 \%$.

Para Pinot noir, la concentración de antocianos fue significativamente mayor para los vinos MRA (10.1\%) elaborados en la vendimia 2016, en cuanto a las restantes vendimias las diferencias entre los tratamientos no fueron significativas. Resultados similares se observaron en los vinos del cultivar Tannat, donde las diferencias entre los tratamientos fueron significativas solo en la vendimia 2017 $(10.7 \%)$. Con la aplicación de esta técnica de reducción de la concentración de alcohol podría esperarse una menor concentración de antocianos ya que se eliminó una porción de mosto. Sin embargo, el reemplazo por mosto de uva verde no implicaría necesariamente pérdidas de antocianos ya que esta operación se realiza antes de la maceración. Por otra parte, el descarte de parte del mosto de uvas maduras durante la sustitución podría implicar una pérdida de antocianos ya que estos se extraen fácilmente de los hollejos durante el proceso de estrujado durante el corto tiempo de contacto con el mosto, por lo que la fracción de mosto eliminada podría contener una cantidad considerable de antocianos [33]. La similar o mayor concentración de antocianos de los vinos MRA en relación con los vinos MT demuestran que este efecto no fue relevante. Asimismo, estos resultados también podrían estar vinculados a la liberación favorecida de copigmentos durante la fermentación, que protege a los antocianos contra la oxidación [34].

La concentración de catequinas no difirió entre los vinos de los distintos tratamientos con la excepción de los vinos Pinot noir elaborados durante la vendimia 2016.
Estos resultados concuerdan con los reportados en otros estudios [20,35].

El efecto de reducción del contenido de alcohol sobre la concentración de proantocianidinas no fue claro. Tanto para Pinot noir como Tannat, se observaron valores significativamente mayores de proantocianidinas en los vinos MRA en una vendimia, menores en otra y sin diferencia con los vinos MT en otra. Sin embargo, Rolle et al. [20] encontraron que en los vinos en los que se redujo el contenido de alcohol sustituyendo mosto de uva madura por mosto de la misma uva tratado por osmosis inversa, los flavonoles de alto peso molecular disminuyeron. La menor concentración de etanol podría dificultar la extracción de flavonoles polimerizados de las uvas durante la fermentación [33]. Por otra parte, el trabajo realizado por Gil et al. [18], donde la desalcoholización de los vinos tintos elaborados con uvas Cabernet Sauvignon, Garnacha y Cariñena se hizo utilizando tecnologías de membrana, no se observaron efecto sobre la concentración de proantocianidinas ni en su grado medio de polimerización.

\subsection{Color de los vinos}

Los parámetros cromáticos de los vinos Tannat y Pinot noir elaborados durante las vendimias 2016, 2017 y 2018 se muestran en la Tabla 4.

El color de los vinos MT y MRA presentó diferencias asociadas al cultivar y al año de vendimia. Como es de esperar, en todas las elaboraciones, los vinos con mayor intensidad colorante fueron los que tuvieron menor luminosidad.

El efecto de la reducción de alcohol sobre el color de los vinos Pinot noir no fue claro. Para la vendimia 2016, los vinos MRA presentaron mayor intensidad colorante respecto de los MT, lo que se explica por una mayor concentración de antocianos. Sin embargo, en las vendimias 2017 y 2018 se observó el efecto contrario, a iguales concentración de antocianos entre tratamientos. Las diferencias en la cromaticidad $\left(\mathrm{C}^{*}\right)$ y el tono $\left(\mathrm{H}^{*}\right)$ entre los vinos MRA y MT fue variable según el año de vendimia.

Sin embargo, los vinos MRA elaborados a partir del cultivar Tannat presentaron una intensidad colorante 
Tabla 3. Composición fenólica de los vinos según cultivar y año de vendimia.

\begin{tabular}{|c|c|c|c|c|c|c|}
\hline Cultivar & $\begin{array}{c}\text { Año de } \\
\text { vendimia }\end{array}$ & $\begin{array}{c}\text { Composición } \\
\text { del mosto }\end{array}$ & $\begin{array}{c}\text { Polifenoles } \\
\text { totales }(\mathrm{mg} / \mathrm{L})\end{array}$ & $\begin{array}{c}\text { Antocianos } \\
(\mathrm{mg} / \mathrm{L})\end{array}$ & $\begin{array}{c}\text { Catequinas } \\
(\mathrm{mg} / \mathrm{L}\end{array}$ & $\begin{array}{c}\text { Proantocianidinas } \\
(\mathrm{mg} / \mathrm{L})\end{array}$ \\
\hline \multirow{6}{*}{ Pinot noir } & 2016 & MT & $1201 \pm 49 a$ & $283 \pm 13 b$ & $742 \pm 38 b$ & $1830 \pm 68 \mathrm{~b}$ \\
\hline & & MRA & $1201 \pm 36 \mathrm{a}$ & $315 \pm 16 \mathrm{a}$ & $840 \pm 64 \mathrm{a}$ & $2075 \pm 110 \mathrm{a}$ \\
\hline & 2017 & MT & $644 \pm 57 \mathrm{a}$ & $151 \pm 7 \mathrm{a}$ & $183 \pm 24 \mathrm{a}$ & $529 \pm 49 \mathrm{a}$ \\
\hline & & MRA & $681 \pm 10 \mathrm{a}$ & $160 \pm 2 \mathrm{a}$ & $176 \pm 46 \mathrm{a}$ & $423 \pm 39 b$ \\
\hline & 2018 & MT & $829 \pm 31 \mathrm{a}$ & $207 \pm 7 \mathrm{a}$ & $536 \pm 10 \mathrm{a}$ & $883 \pm 29 a$ \\
\hline & & MRA & $795 \pm 37 \mathrm{a}$ & $190 \pm 9 a$ & $504 \pm 32 \mathrm{a}$ & $930 \pm 68 \mathrm{a}$ \\
\hline \multirow{6}{*}{ Tannat } & 2016 & MT & $2376 \pm 126 \mathrm{a}$ & $1031 \pm 52 \mathrm{a}$ & $1408 \pm 31 \mathrm{a}$ & $3711 \pm 107 \mathrm{a}$ \\
\hline & & MRA & $2155 \pm 89 b$ & $1010 \pm 67 \mathrm{a}$ & $1328 \pm 78 \mathrm{a}$ & $3454 \pm 113 b$ \\
\hline & 2017 & MT & $1311 \pm 20 \mathrm{a}$ & $523.8 \pm 6 b$ & $1058 \pm 43 \mathrm{a}$ & $1965 \pm 42 b$ \\
\hline & & MRA & $1367 \pm 07 \mathrm{a}$ & $586.1 \pm 26 \mathrm{a}$ & $1125 \pm 54 \mathrm{a}$ & $2142 \pm 15 \mathrm{a}$ \\
\hline & 2018 & MT & $1773 \pm 42 \mathrm{a}$ & $941 \pm 32 \mathrm{a}$ & $1351 \pm 182 \mathrm{a}$ & $2700 \pm 57 \mathrm{a}$ \\
\hline & & MRA & $1718 \pm 43 \mathrm{a}$ & $932 \pm 28 \mathrm{a}$ & $1411 \pm 71 \mathrm{a}$ & $2609 \pm 109 \mathrm{a}$ \\
\hline
\end{tabular}

Medias con distinta letra indican diferencias significativas $(p<0.05)$. MT: vino elaborado con mosto testigo; MRA: vino elaborado con mosto reducido en azúcares.

Tabla 4. Color de los vinos segn cultivar y año de vendimia.

\begin{tabular}{|c|c|c|c|c|c|c|}
\hline Cultivar & Año de vendimia & Composición del mosto & Intensidad colorante & $\mathbf{L}^{*}$ & $\mathbf{C}^{*}$ & $\mathbf{H}^{*}$ \\
\hline \multirow{6}{*}{ Pinot noir } & 2016 & MT & $11.0 \pm 0.4 \mathrm{~b}$ & $70.9 \pm 0.9 \mathrm{a}$ & $20.7 \pm 0.8 b$ & $17.5 \pm 1.8 \mathrm{~b}$ \\
\hline & & MRA & $13.0 \pm 0.3 \mathrm{a}$ & $63.2 \pm 0.8 \mathrm{~b}$ & $31.2 \pm 0.3 \mathrm{a}$ & $355.0 \pm 0.7 \mathrm{a}$ \\
\hline & 2017 & MT & $6.4 \pm 0.2 \mathrm{a}$ & $72.3 \pm 1.4 \mathrm{a}$ & $25.2 \pm 1.4 \mathrm{a}$ & $27.2 \pm 1.5 \mathrm{a}$ \\
\hline & & MRA & $5.9 \pm 0.4 b$ & $73.2 \pm 5.2 \mathrm{a}$ & $16.9 \pm 1.9 \mathrm{~b}$ & $24.8 \pm 1.2 \mathrm{~b}$ \\
\hline & 2018 & MT & $4.6 \pm 0.1 \mathrm{a}$ & $76.7 \pm 0.7 \mathrm{a}$ & $23.9 \pm 0.4 \mathrm{a}$ & $26.6 \pm 0.1 \mathrm{a}$ \\
\hline & & MRA & $4.0 \pm 0.1 \mathrm{~b}$ & $79.2 \pm 1.1 \mathrm{a}$ & $22.7 \pm 0.7 \mathrm{a}$ & $20.7 \pm 1.3 \mathrm{~b}$ \\
\hline \multirow{6}{*}{ Tannat } & \multirow[t]{2}{*}{2016} & MT & $26.7 \pm 0.9 \mathrm{~b}$ & $38.6 \pm 1.3 \mathrm{a}$ & $43.8 \pm 0.5 \mathrm{a}$ & $348.1 \pm 1.0 \mathrm{a}$ \\
\hline & & MRA & $29.9 \pm 2.1 \mathrm{a}$ & $34.8 \pm 1.7 \mathrm{~b}$ & $45.6 \pm 4.2 \mathrm{a}$ & $348.9 \pm 1.8 \mathrm{a}$ \\
\hline & \multirow[t]{2}{*}{2017} & MT & $12.7 \pm 0.5 \mathrm{~b}$ & $67.3 \pm 1.1 \mathrm{a}$ & $22.0 \pm 0.5 b$ & $14.8 \pm 0.7 \mathrm{a}$ \\
\hline & & MRA & $14.2 \pm 0.2 \mathrm{a}$ & $63.8 \pm 0.5 \mathrm{~b}$ & $25.4 \pm 0.7 \mathrm{a}$ & $12.7 \pm 1.6 \mathrm{~b}$ \\
\hline & \multirow[t]{2}{*}{2018} & MT & $19.6 \pm 0.7 \mathrm{a}$ & $31.7 \pm 1.0 \mathrm{a}$ & $55.3 \pm 0.1 \mathrm{a}$ & $12.4 \pm 0.6 \mathrm{a}$ \\
\hline & & MRA & $19.3 \pm 0.6 \mathrm{a}$ & $32.6 \pm 0.8 \mathrm{a}$ & $56.7 \pm 0.3 \mathrm{a}$ & $11.0 \pm 0.2 \mathrm{~b}$ \\
\hline
\end{tabular}

Medias con distinta letra indican diferencias significativas $(p<0.05)$. $\mathrm{L}^{*}$ : luminosidad; $\mathrm{C}^{*}$ : cromaticidad; $\mathrm{H}^{*}$ : tono; MT: vino elaborado con mosto testigo; MRA: vino elaborado con mosto reducido en azúcares.

significativamente superior a los MT. La cromaticidad y el tono de los vinos MRA fueron iguales o superiores a los MT. Estos resultados pueden estar asociados a las diferencias en el $\mathrm{pH}$ de los vinos. Como es sabido, cuando el $\mathrm{pH}$ disminuye, el equilibrio entre las diferentes formas de los antocianos se desplaza hacia la forma roja de las moléculas, el catión flavilio [36]. Otro factor que puede incidir es la contribución de los copigmentos al color del vino. La copigmentación es un fenómeno importante, que ocurre en los vinos tintos jóvenes. El etanol tiene un papel disociador en los complejos de copigmentación como consecuencia del debilitamiento de las interacciones hidrofóbicas [37]. Sin embargo, la solubilidad de algunos copigmentos podría aumentar con la mayor producción de etanol, compensando el efecto disruptivo. La matriz del vino influye en el efecto del etanol sobre la copigmentación y el color, particularmente la proporción entre antocianos y copigmentos relacionados [34].

Se debe considerar que los cultivares Tannat y Pinot noir presentan diferencias considerables en su composición fenólica, lo cual podría explicar las diferencias observadas en los resultados. Tannat se caracteriza por tener contenidos muy elevados de pigmentos (antocianos) y taninos, en cuanto Pinot noir presenta una gran riqueza polifenólica (sobre todo en semillas), pero constituida casi exclusivamente por taninos, con muy bajos contenidos de antocianos.

\subsection{Análisis multivariado de la varianza}

Los análisis multivariados de la varianza muestran el efecto de cada factor en los diferentes componentes de los vinos (Tabla 5). Se verifica una incidencia muy importante del año de vendimia, la composición del mosto y su interacción sobre el contenido de alcohol, el pH y la acidez titulable de los vinos Pinot noir. Para el caso de los vinos Tannat, la mayor incidencia de estos factores se observó en el contenido de alcohol y en el $\mathrm{pH}$, la acidez titulable fue afectada por el año de vendimia. Estos resultados parecen lógicos ya que la composición del mosto en cosecha (concentración de azúcares, pH y acidez titulable) fue muy diferente entre añadas (Tabla 1). Además, los tratamientos donde se realizó la sustitución de mosto modifican la composición de este y en consecuencia del vino. 
Tabla 5. Análisis multifactorial de la varianza.

\begin{tabular}{|c|c|c|c|c|}
\hline Cultivar & $\begin{array}{l}\text { Parámetros } \\
\text { analíticos }\end{array}$ & $\begin{array}{c}\text { Año de } \\
\text { vendimia }\end{array}$ & $\begin{array}{c}\text { Composición del } \\
\text { mosto }\end{array}$ & $\begin{array}{c}\text { Año de vendimia } x \\
\text { Composición del mosto }\end{array}$ \\
\hline \multirow{11}{*}{ Pinot noir } & Alcohol & $757.97 * * *$ & $1848.09 * * *$ & $142.18 * * *$ \\
\hline & Acidez total & $1196.57 * * *$ & $940.21 * * *$ & $687.74 * * *$ \\
\hline & pH & $752.53 * * *$ & $1258.73 * * *$ & $195.58 * * *$ \\
\hline & Polifenoles totales & $546.32 * * *$ & 1.44 & 0.51 \\
\hline & Antocianos & $629.62 * * *$ & $6.18 *$ & $17.28 * * *$ \\
\hline & Catequinas & $717.39 * * *$ & 2.31 & $9.20 * * *$ \\
\hline & Proantocianidinas & $1573.83 * * *$ & $8.08 * *$ & $20.86 * * *$ \\
\hline & Intensidad colorante & $1435.56 * * *$ & $7.77 * *$ & $48.05 * * *$ \\
\hline & $\mathbf{L}^{*}$ & $66.88 * * *$ & $3.39 *$ & $17.09 * * *$ \\
\hline & $\mathbf{C}^{*}$ & $61.11 * * *$ & 0.87 & $230.39 * * *$ \\
\hline & $\mathbf{H}^{*}$ & $930.77 * * *$ & $630.50 * * *$ & $233.88 * * *$ \\
\hline \multirow{11}{*}{ Tannat } & Alcohol & $1711.41 * * *$ & $699.08 * * *$ & $79.76 * * *$ \\
\hline & Acidez total & $36.33 * * *$ & $4.94 *$ & 2.46 \\
\hline & pH & $9.54 * * *$ & $24.14 * * *$ & $7.67 * *$ \\
\hline & Polifenoles totales & $477.14 * * *$ & $9.02 * *$ & $10.77 * * *$ \\
\hline & Antocianos & $456.07 * * *$ & 0.63 & $3.75 *$ \\
\hline & Catequinas & $38.53 * * *$ & 0.27 & 2.45 \\
\hline & Proantocianidinas & $586.31 * * *$ & 2.4 & $11.89 * * *$ \\
\hline & Intensidad colorante & $638.70 * * *$ & $18.96 * * *$ & $8.81 * *$ \\
\hline & $\mathbf{L}^{*}$ & $3045.80 * * *$ & $3097 * * *$ & $16.33 * * *$ \\
\hline & $\mathbf{C}^{*}$ & $1026.31 * * *$ & $13.79 * * *$ & 1.07 \\
\hline & $\mathbf{H}^{*}$ & $1865.48 * * *$ & $5.72 *$ & $5.31 *$ \\
\hline
\end{tabular}

Se indican los valores de $\mathrm{F}$ y su significación estadística $(p<0.001=* * * ; p<0.01=* * ; p<0.1=*$ ).

El año de vendimia influyó fuertemente en la concentración de todos los compuestos fenólicos. Sin embargo, el efecto de la composición del mosto fue menos significativo. La interacción año de vendimia composición del mosto fue más significativa para los vinos Pinot noir que para los vinos Tannat. Varios autores han demostrado que la composición fenólica de la uva y, por lo tanto, del vino está determinada por las condiciones de maduración de cada año en particular [24,32]. La composición del mosto solo influyó significativamente en la concentración de polifenoles totales. Como se discutió anteriormente, el contenido de etanol y el $\mathrm{pH}$ son factores que contribuyen a la extracción durante la maceración fermentativa.

El año de vendimia tuvo un fuerte impacto sobre todos los parámetros cromáticos de los vinos de ambos cultivares. Por su parte la composición del mosto tuvo un impacto diferente de acuerdo con el cultivar. Para los vinos Tannat, la composición del mosto tuvo un impacto muy relevante en la intensidad colorante, la luminosidad (L*) y cromaticidad $\left(\mathrm{C}^{*}\right)$, en cuanto al tono $\left(\mathrm{H}^{*}\right)$ fue menos afectado. Para los vinos Pinot noir el mayor impacto se observó en el tono $\left(\mathrm{H}^{*}\right)$ y en la intensidad colorante. La interacción año de vendimia - composición del mosto fue altamente significativa para todos los parámetros cromáticos determinados en los vinos Pinot noir, en cambio que para los vinos Tannat solo fue relevante en la luminosidad e intensidad del color. El color del vino tinto es el resultado de la concentración de pigmentos, sus interacciones con otros compuestos y las condiciones físico-químicas del medio en el que se encuentran. Por lo tanto, cualquier modificación de estos factores determina un cambio en el color del vino.

\section{Conclusiones}

Se puede concluir que la técnica de sustitución de una proporción de mosto de uva madura por mosto de uva inmadura permitió elaborar vinos Tannat y Pinot noir con menor contenido de alcohol y el pH.

La sustitución de mosto no redujo la concentración de polifenoles totales, catequinas y antocianos en los vinos de ambos cultivares. El efecto sobre la concentración de proantocianidinas fue variable de acuerdo con el cultivar y con el año de vendimia.

El efecto de la sustitución de mosto sobre el color dependió del cultivar. Los vinos Tannat elaborados por este tratamiento tuvieron una mayor intensidad de color con menor tono e igual cromaticidad que los vinos testigos. En los vinos Pinot noir las tendencias no fueron claras.

El año de vendimia tuvo un impacto muy importante en la composición y el color de los vinos en tanto que la composición del mosto impactó más fuertemente en la composición básica y el color con un efecto diverso según el cultivar.

Los autores agradecen al Instituto Nacional de Vitivinicultura (I.NA.VI.), Establecimiento Juanicó y Bodega Olga Silva.

El trabajo contó con el apoyo financiero de la CAP (Comisión Académica de Posgrado de la Universidad de la República), ANII (Agencia Nacional de Investigación e Innovación, MOV_CA_2015_1_107599) y CISC (Comisión Sectorial de Investigación Científica, beca de Movilidad, 2016 y 2017). 


\section{Referencias}

[1] J. Ó-Marques, R. Reguinga, O. Laureano, J. Ricardoda-Silva, Ciência e Técnica Vitivinicola 20, 35 (2005)

[2] M. Gil, N. Kontoudakis, E. Gonzailez, M. Esteruelas, F. Fort, J.M. Canals, F. Zamora, Agric. Food Chem. 60, 7988 (2012)

[3] H. Fulcrand, H., M. Dueñas, E. Salas, V. Cheynier, Am. J. Enol. Vitic 57, 289 (2006)

[4] K. Sacchi, L. Bisson, D. Adams, Am. J. Enol. Vitic. 56, 197 (2005)

[5] F.W. Beech, L.F. Burroughs, C.F. Timberlake, G.C. Bull, OIV 52, 1001 (1979)

[6] G.V. Jones, M.A. White, O.R. Cooper, Clim. Change 73, 319 (2005)

[7] R. Mira de Orduña, Food Res. Int. 43, 1844 (2010)

[8] H. Yu, O. Lee, J. Lee, S. Choi, J. Lee, Food Sci. Biotech. 14, 772 (2005)

[9] G. González-Neves, D. Charamelo, J. Balado, L. Barreiro, R. Bochicchio, G. Gatto, G. Gil, A. Tessore, A. Carbonneau, M. Moutounet, Anal. Chim Acta 513, 191 (2004)

[10] J. Labanda, S. Vichi, J. Llorens, E. Lopez-Tamames, Food Sci. and Technol. 42, 1390 (2009)

[11] L.M. Schmidtke, J. W. Blackman, S.O. Agboola, J. Food Sci. 77, 25 (2012)

[12] G.J. Pickering, J. Wine Res. 11, 129 (2000)

[13] H.R. Schultz, Aust. J. Grape Wine Res. 6, 2 (2000)

[14] G.J. Pickering, D.A. Heatherbell, M.F. Barnes, Food Res. 31, 685 (1998)

[15] M. Ciani, L. Ferraro, Microbiology 62, 128 (1996)

[16] L. Takács, G. Vataim, K. Korány, J. Food Eng. 78, 118 (2007)

[17] N. García-Martín, S. Pérez-Magariño, M. OrtegaHeras, C. González-Huertas, M. Mihnea, M. González-Sanjosé, L. Palacio, P. Prádanos, A. Hernández, Sep. Purif. Technol. 76, 158 (2010)

[18] M. Gil, S. Estevez, N. Kontoudakis, F. Fort, J.M. Canals, F. Zamora, Eur. Food Res. Technol. 237, 481 (2013)
[19] N. Kontoudakis, M. Esteruelas, F. Fort, J.M. Canals, V. De Freitas, F. Zamora, Food Chem. 124, 767 (2011)

[20] L. Rolle, V. Englezos, F. Torchio, F. Cravero, S. Río Segade, K. Rantsiou, S. Giacosa, A. Gambuti, V. Gerbi, L. Cocolin, Aust. J. Grape Wine Res. 24, 62 (2018)

[21] Vitis International variety Catalogue, http://www.vivc.de/

[22] OIV. Vol. 1. Paris, France: Organisation Internationale de la Vigne et du Vin (2018)

[23] Y. Glories, M. Augustin, In C.R. Colloque Journée Techn. CIVB (Bordeaux, 1993), p. 56

[24] G. González-Neves, L. Barreiro, G. Gil, J. Franco, A. Carbonneau, M. Moutounet, Bull. OIV. 887, 30 (2005)

[25] Y. Glories, Conn. Vigne Vin 18, 195 (1984)

[26] F. Ayala, J.F. Echávarri, A.I. Negueruela, Am. J. Enol. Vitic. 48, 357 (1997)

[27] F. Ayala, J.F. Echávarri, A.I. Negueruela. MSCVes.zip, URL http://www.unizar.es/ negueruela/MSCV.es (2001)

[28] V. Singleton, J. Rossi, Am. J. Enol. Vitic. 16, 144 (1965)

[29] P. Ribéreau-Gayon, E. Stonestreet, Chim. Anal. 48, 188 (1966)

[30] T. Swain, W. Hillis, J. Sci. Food Agric. 10, 63 (1959)

[31] J.A. Di Rienzo, F. Casanoves, M.G. Balzarini, L. Gonzalez, M. Tablada, C.W Robledo, InfoStat versión 2015. Grupo InfoStat, FCA, Universidad Nacional de Córdoba, Argentina. URL http://www.infostat.com.ar

[32] M. Ferrer, G. Echeverría, M. Miras-Avalos, IJOEAR 8, 16 (2017)

[33] R. Canals, M.C. Llaudy, J. Valls, J.M. Canals, F. Zamora, J. Agric. Food Chem. 53, 4019 (2005)

[34] R. Boulton, Am. J. Enol. Vitic. 52, 67 (2001)

[35] A. Gambuti, A. Rinaldi, M.T. Lisanti, R. Pessina, L. Moio, Eur. Food Res. Technol. 233, 647 (2011)

[36] F. He, N. Liang, L. Mu, Q. Pan, J. Wang, J. Reeves, C. Duan, Moléculas 17, 1571 (2012)

[37] I. Hermosín Gutiérrez, J. Agric. Food Chem. 514079 (2003) 